\title{
Integrated Image Registration for Cardiac MR Perfusion Data
}

\author{
Ravi Bansal ${ }^{1}$ and Gareth Funka-Lea ${ }^{1}$ \\ Department of Imaging and Visualization, Siemens Corporate Research, Inc. \\ Princeton, NJ 08540
}

\begin{abstract}
In this paper we present an integrated image registration algorithm for segmenting the heart muscle, the myocardium (MC). A sequence of magnetic resonance (MR) images of heart are acquired after injection of a contrast agent. An analysis of the perfusion of the contrast agent into myocardium is utilized to study its viability. Such a study requires segmentation of $\mathrm{MC}$ in each of the images acquired which is a difficult task due to rapidly changing contrast image the images. In this paper we present an information theoretic registration framework which integrates two channels of information, the pixel intensities and the local gradient information, to reliably and accurately segment the myocardium. In our framework, the physician hand draws contours representing the inner (the endocardium) and the outer (the epicardium) boundaries of the myocardium. These hand drawn contours are then propagated to the other images in the sequence of images acquired to segment the MC.
\end{abstract}

\section{Introduction}

Ischemic heart disease, the obstruction of blood flow to the heart, results due to excess fat or plaque deposits which narrows the veins that supply oxygenated blood to the heart. The reduced blood supply to the heart is typically manifested as reduced blood perfusion to the myocardium (MC), the heart muscle. Clinically, the myocardial perfusion measurements are routinely performed with single-photon emission computed tomography (SPECT) or with positron emission tomography (PET) images [1. Some of the major drawbacks of these techniques are the low spatial resolution, attenuation artifacts of SPECT and limited availability of PET [11,12. Authors in 8,15] show possibility of myocardial perfusion analysis with magnetic resonance images (MRIs) which also permits quantitative analysis of blood flow.

In MR perfusion analysis study, usually about 60 to 100 short axis 2D MR images of the heart are acquired after injecting contrast agent into the blood. As the heart is beating, the contrast in the acquired MR images is rapidly changing. The contrast agent passes through right ventricle (RV) to left ventricle (LV) and then perfuses into the myocardium. To do the perfusion analysis, it is necessary to segment the myocardium in all the MR images acquired in a perfusion scan. This is a tedious job, given that there are 60 to 100 images in each scan. The 
problem gets compounded due to the fact that the contrast in the images is rapidly changing. When the contrast agent is in LV, the blood pool brighten ups and makes it easy to segment the inner wall of the myocardium, the endocardium. However, when there is no contrast agent in LV, it is very difficult to segment the endocardium. Segmentation of the outer boundary of the heart, the epicardium, remains difficult throughout the images acquired in the scan. In addition to the changing contrast, there could also be gross motion due to patient breathing and changes in heart shape as it is beating. Only recently there has been some published work 2,13] in the medical image processing literature to address these issues.

\section{Our Approach}

In this paper we pose the myocardium segmentation problem as an image registration problem. Segmentation is achieved by template matching. In our registration framework, a physician hand draws contours, denoting the epicardium and endocardium, on one of the 2D MR images in the sequence. These contours are used to define a region of interest (ROI) locally around the hand drawn contours. This ROI, or the template, is then correlated with other images in the sequence to best estimate the myocardium.

As the image contrast is changing rapidly, we decided to utilize mutual information (MI) based match criteria 314 for template matching while assuming only whole pixel shifts. However, in the images, there could be sub-pixel shifts which can quickly add up to a large motion if we utilize only gray scale information. Thus, there was a necessity to incorporate edge information within the registration framework to better estimate the registration parameters. Due to rapidly changing contrast in the images, sometimes the epicardium and the endocardium are not visible. In these cases then in order to keep propagating the contours properly, it is essential to utilize gray scale information. In this paper we present an example where while trying to achieve sub-pixel accuracy, in MI based approach, by doing bilinear interpolation leads to worse estimate of the registration parameters. Thus, we decided to limit estimation of the registration parameters to whole pixel shifts only while achieving further accuracy using the edge information. A number of methods have been proposed within image registration literature [59|10] which also combine gray scale with edge information to better estimate registration parameters. However, we feel that while these methods represent significant advances, they remain ad-hoc in their approach.

\subsection{Mathematical Formulation}

In our registration framework, the image on which the contours are hand drawn, or where the contours are estimated in the previous iteration, is called the template image. The image where the contours are being currently propagated to is called the current image. Our registration framework consists of two steps. In the first step we estimate the probability of each pixel, in the current image, being 
an edge as a function of local gradient and location of the contours at the current estimate of the registration parameters. These estimated edge probabilities are then utilized in the second step to estimate the registration parameters. These two steps are repeated till convergence. In our current formulation, we do not address the problem of changing heart shape, an issue that we plan to address in our future work.

Estimating Edge Probabilities. To estimate the edge probabilities in the current image, we model the current image as a 2D MRF (Markov random field) [4] with discontinuities.

Let $S=\{1, \ldots, m\}$ denote the discrete set of $m$ sites on a $2 \mathrm{D}$ grid of the current image. Let $N=\left\{N_{i} \mid \forall i \in S\right\}$ denote the neighborhood system with the properties (1) $i \notin N_{i}$ and (2) $i \in N_{i^{\prime}} \Leftrightarrow i^{\prime} \in N_{i}$. Let the set of first order $C_{1}$ and the set of second order $C_{2}$ cliques be defined as $C_{1}=\{i \mid \forall i \in S\}$ and $C_{2}=\left\{\left\{i, i^{\prime}\right\} \mid \forall i \in S, i^{\prime} \in N_{i}\right\}$. Let $F=\left\{F_{1}, \ldots, F_{m}\right\}$ be a family of random variables which are defined on $S$ and let $f=\left\{f_{1}, \ldots, f_{m}\right\}$ be a realization of $F$. Define an energy function $U(f)$ as a function of the clique potentials $V_{c}(f)$ to be $U(f)=\sum_{c \in C} V_{c}(f)$.

Then the Gibbs distribution of the random field $F$ is defined as $P(f)=\frac{1}{Z}$ $\exp (-\beta U(f))$ which is also the probability density function (pdf) on the MRF [4]7. $Z$, also called the partition function, is a normalization constant. Let $e_{i i^{\prime}}^{2}$ be a random variable denoting an edge between sites $i$ and $i^{\prime}$ and let $\mathcal{E}_{2}=\left\{e_{i i^{\prime}}^{2} \mid \forall i \in\right.$ $\left.S, i^{\prime} \in N_{i}\right\}$ denote the set of edges. Let $d=\left\{d_{i} \mid \forall i \in S\right\}$ denote the observed data. Let $\sigma$ denote the standard deviation of the noise in the observed data $d$. Let $g_{i i^{\prime}}^{2}$ denote the local intensity gradient in the current image at the site $i$. Let $\mathcal{E}_{1}=\left\{e_{i}^{1} \mid e_{i}^{1} \bowtie e_{i i^{\prime}}^{2} \forall i^{\prime} \in N_{i}, e_{i}^{1} \in C\right\}$ denote the set of corresponding edges, $e_{i}^{1}$, on the contours $C$ in the template image. The symbol $\bowtie$ is used to denote the corresponding edges on the contours $C$. In our formulation, corresponding edges are the edges with the shortest Euclidean distance. Let us denote the distance between the corresponding edges, $e_{i}^{1}$ and $e_{i i^{\prime}}^{2}$ by $s_{i}^{1}$. Finally, let $\mathcal{L}\left(g_{i i^{\prime}}^{2}, s_{i}^{1}\right)$ denote the likelihood of an edge $e_{i i^{\prime}}^{2}$ which is a function of local image gradient and distance to the corresponding edge on the contours $C$.

Using these notations, the energy function, for a second order neighborhood, under given information is written as:

$$
\begin{aligned}
E\left(f, \mathcal{E}_{2}\right) & =U\left(f, \mathcal{E}_{2} \mid d, \mathcal{E}_{1}\right) \\
& =\sum_{i=1}^{m}\left(f_{i}-d_{i}\right)^{2} /\left(2 \sigma^{2}\right)+\sum_{i} \sum_{i^{\prime} \in N_{i}}\left\{\mathcal{L}\left(g_{i i^{\prime}}^{2}, s_{i}^{1}\right)\left(1-e_{i i^{\prime}}^{2}\right)+\alpha e_{i i^{\prime}}^{2}\right\} \\
& =\sum_{i=1}^{m}\left(f_{i}-d_{i}\right)^{2}+\lambda \sum_{i} \sum_{i^{\prime} \in N_{i}}\left\{\mathcal{L}\left(g_{i i^{\prime}}^{2}, s_{i}^{1}\right)\left(1-e_{i i^{\prime}}^{2}\right)+\alpha e_{i i^{\prime}}^{2}\right\} .
\end{aligned}
$$

The likelihood term $\mathcal{L}\left(g_{i i^{\prime}}^{2}, s_{i}^{1}\right)$ is evaluated as:

$$
\mathcal{L}\left(g_{i i^{\prime}}^{2}, s_{i}^{1}\right)=P\left(e_{i}^{1}, g_{i i^{\prime}}^{2} \mid e_{i i^{\prime}}^{2}=1\right)=P\left(e_{i}^{1} \mid e_{i i^{\prime}}^{2}=1\right) P\left(g_{i i^{\prime}}^{2} \mid e_{i i^{\prime}}^{2}=1\right),
$$

where we assume that $e_{i}^{1}$ and $g_{i i^{\prime}}^{2}$ are conditionally independent random variables and $P\left(e_{i}^{1} \mid e_{i i^{\prime}}^{2}=1\right)$ is evaluated as a function of $s_{i}^{1}$. The Gibbs distribution of the random field is then given as $P^{G b}\left(E\left(f, \mathcal{E}_{2}\right)\right)=\frac{1}{Z} \exp \left\{-\beta E\left(f, \mathcal{E}_{2}\right)\right\}$.

The energy $E\left(f, \mathcal{E}_{2}\right)$ can then be optimized to estimate the MAP (maximum a-posteriori) estimate of the random field. However, this is a classical optimization problem which involved both discrete and continuous random variables. To 
overcome this problem, $\mathcal{E}_{2}$ are usually approximated with continuous variables [6]16]. However, in this paper we propose to integrate-out the edge variables. In the process of integrating-out the edge variables, a new set of variables, $l_{i i^{\prime}}$, appear which can be shown to be probability of not observing an edge given all the information. That is, $P^{G b}(E(f))=\sum_{\mathcal{E}_{2}} P^{G b}\left(E\left(f, \mathcal{E}_{2}\right)\right)$, where after few steps it can be shown that

. $\quad E(f)=\sum_{i=1}^{m}\left(f_{i}-d_{i}\right)^{2}+\lambda \sum_{i} \sum_{i^{\prime} \in N_{i}}\left\{\mathcal{L}\left(g_{i i^{\prime}}^{2}, s_{i}^{1}\right)+\ln l_{i i^{\prime}}\right\}$

with $l_{i i^{\prime}}=\frac{1}{1+\exp \left\{-\beta \lambda\left(\mathcal{L}\left(g_{i i^{\prime}}^{2} s_{i}^{1}\right)-\alpha\right)\right\}}=P\left(e_{i i^{\prime}}^{2}=0 \mid f, \mathcal{E}_{2}\right)$. The estimated $l_{i i^{\prime}}$ are then utilized on the next step to better estimate the registration parameters.

Estimating Registration Parameters. Let $Y=\left\{Y_{1}, \ldots, Y_{m}\right\}$ be a random field denoting pixel intensities of the template image. Let $y=\left\{y_{1}, \ldots, y_{m}\right\}$ be a particular realization. Let $T$ denote the two translation parameters that are being estimated. Then the optimal registration parameters, $\hat{T}$, are being estimated as minimization of the joint conditional entropy

$$
\begin{aligned}
\hat{T} & =\arg \min _{T} H\left(F, \mathcal{E}_{2} \mid Y, \mathcal{E}_{1}, T\right)=\arg \min _{T} H\left(F, \mathcal{E}_{2} \mid Y(T), \mathcal{E}_{1}(T)\right) \\
& =\arg \min _{T}\left[H\left(F \mid \mathcal{E}_{2}, Y(T), \mathcal{E}_{1}(T)\right)+H\left(\mathcal{E}_{2} \mid Y(T), \mathcal{E}_{1}(T)\right)\right] \\
& \leq \arg \min _{T}\left[H(F \mid Y(T))+H\left(\mathcal{E}_{2} \mid \mathcal{E}_{1}(T)\right)\right]=\arg \min _{T}\left[H(F \mid Y(T))+H\left(\mathcal{E}_{2}, \mathcal{E}_{1}(T)\right)\right],
\end{aligned}
$$

where $H\left(\mathcal{E}_{1}, T\right)$ is assumed to be a constant and $H(x)=-\sum p(x) \log p(x)$ is the Shannon's entropy. The first term in the equation above is the conditional entropy which is similar to the gray scale conditional entropy term in the mutual information formulation. The second term minimizes the entropy of the estimated edges in the current image and the edges on the contours in the template image. Thus, the above formulation integrates the two channels of information to better estimate the registration parameters.

To formulate the problem within the entropy framework, joint entropy between $\mathcal{E}_{2}$ and $\mathcal{E}_{1}$ is approximated as a joint entropy between $\mathcal{E}_{2}$ and the distance transform $S(T)$ of $\mathcal{E}_{1}$. This assumption is based on the intuition that when the two images are registered, the entropy of the distribution of the distances under $\mathcal{E}_{2}$ will be minimal. Thus, we evaluate $H\left(\mathcal{E}_{2}, \mathcal{E}_{1}(T)\right)=H\left(\mathcal{E}_{2}, S(T)\right)$.

We assume the each pixel is independently distributed and hence write the joint distribution $p\left(\mathcal{E}_{2}, S(T)\right)$ as:

$$
p\left(\mathcal{E}_{2}, S(T)\right)=\prod_{i} \prod_{i^{\prime} \in N_{i}} p\left(e_{i i^{\prime}}^{2}, s_{i}\right)=\prod_{i} \prod_{i^{\prime} \in N_{i}} P\left(e_{i i^{\prime}}^{2}\right) p\left(s_{i} \mid e_{i i^{\prime}}^{2}\right) .
$$

Thus, the joint entropy $H\left(\mathcal{E}_{2}, S(T)\right)$ can be written as

$$
H\left(\mathcal{E}_{2}, S(T)\right)=\sum_{i} \sum_{i^{\prime} \in N_{i}} P\left(e_{i i^{\prime}}^{2}=1\right) H_{i 1}(s)+\sum_{i} \sum_{i^{\prime} \in N_{i}} P\left(e_{i i^{\prime}}^{2}=0\right) H_{i 0}(s)
$$

where $H_{i 1}(s)=-\sum p\left(s_{i} \mid e_{i i^{\prime}}^{2}=1\right) \log p\left(s_{i} \mid e_{i i^{\prime}}^{2}=1\right)$. Further assuming that $H_{i 1}(s)$ is identically distributed for each $i$ and assuming that $H_{i 0}(s)$ is almost constant, the joint entropy $H\left(\mathcal{E}_{2}, S(T)\right)$ is further approximated to be

$$
\begin{aligned}
H\left(\mathcal{E}_{2}, S(T)\right) & =\sum_{i} \sum_{i^{\prime} \in N_{i}} P\left(e_{i i^{\prime}}^{2}=1\right) H_{i 1}(s)=H_{1}(s) \sum_{i} \sum_{i^{\prime} \in N_{i}} P\left(e_{i i^{\prime}}^{2}=1\right) \\
& =N H_{1}(s) \frac{\sum_{i} \sum_{i^{\prime} \in N_{i}} P\left(e_{i i^{\prime}}^{2}=1\right)}{N}=N<\bar{e}>H_{1}(s)
\end{aligned}
$$

Thus, finally, under the i.i.d. assumption, the optimal transformation parameters are estimated as

$$
\begin{aligned}
\hat{T} & =\arg \min _{T}\left[H(F \mid Y(T))+H\left(\mathcal{E}_{2}, \mathcal{E}_{1}(T)\right)\right] \\
& =\arg \min _{T}\left[m H(f \mid y(T))+N<\bar{e}>H_{1}(s)\right]
\end{aligned}
$$




\subsection{Current Implementation}

In our current implementation we further assume that the edges are localized at the pixels rather that between two pixels. Under this simplifying assumption, the two steps are

\section{Estimating Edge Probabilities.}

$$
l_{i}=\frac{1}{1+\exp \left\{-\beta \lambda\left(\mathcal{L}\left(g_{i}^{2}, s_{i}^{1}\right)-\alpha\right)\right\}},
$$

where $g_{i}^{2}$ denotes the local gradient magnitude at the site $i$ and $l_{i}$ denotes the probability of no edge at site $i$.

Estimating the Registration Parameters. Once the edge probabilities are estimated in the previous step, the optimal registration parameters $\hat{T}$ are estimated as

$$
\hat{T}=\arg \min _{T}\left[H(f \mid y)+<\bar{e}>H_{1}(s)\right],
$$

where $\langle\bar{e}\rangle=\frac{\sum_{i} P\left(e_{i}^{2}=1\right)}{m}$.

The algorithm is initialized with all edge probabilities set to zero and initialize the temperature $1 / \beta$ to a high value. Then the algorithm estimates the registration parameters (eq. (2) ), updates the edge probabilities (eq. (1)), decreases the temperature and repeats till convergence.

\section{Results}

In this section we present the results of our integrated registration framework. In the results presented in this paper, we are estimating only two translation parameters. Thus, we are assuming that there is no rotation. Also, we assume that there are no sub-pixel shifts. This assumption is not true and might lead to drifts in estimated registration as the sub-pixel shifts add-up.

Fig. 11 shows few frames from a sequence of frames of synthetic data where, in addition to rapidly changing pixel intensities, there is a sub-pixel shift to the right. First row of images show the results obtained using mutual information 143] based template matching where we assume no sub-pixel shifts. The registration is being done using pixel intensities only and the hand drawn contours are utilized to specify the ROI only. Notice that under this assumption the sub-pixel drifts quickly add up leading to noticeably shift towards the end of sequence.

Fig.2 shows results obtained for the MR perfusion study for a heavily sedated dog. As the dog is heavily sedated, no motion is expected. The first row of images in Fig,2] show the results obtained using pixel intensity based mutual information based registration algorithm. Again we are estimating only whole pixel shifts. Even though we are estimating only whole pixel shifts, we see that visually the mutual information based strategy accurately localized the myocardium. 

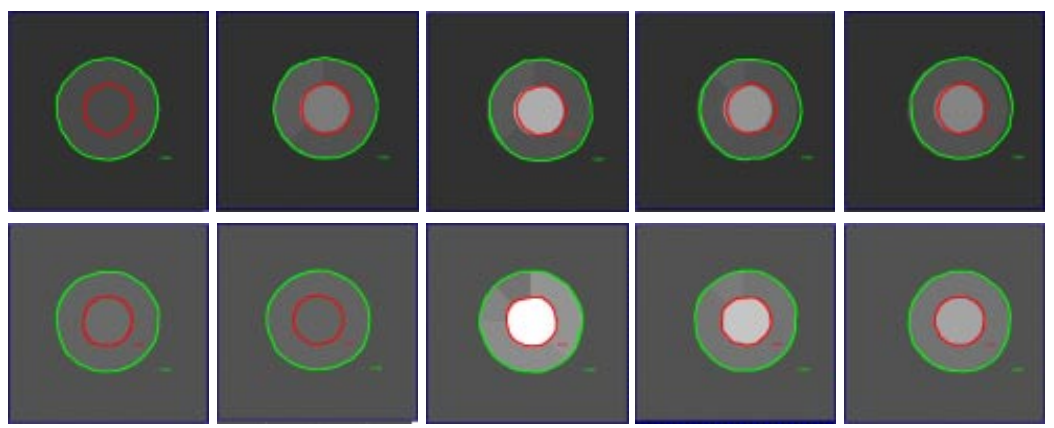

Fig. 1. Selected images from a sequence of synthetic data with sub-pixel shifts, in addition to rapidly changing pixel intensities. First row of images show results obtained using gray scale based mutual information only assuming no sub-pixel shifts. The second row of images show the results obtained using our proposed algorithm while estimating only whole pixel shifts.
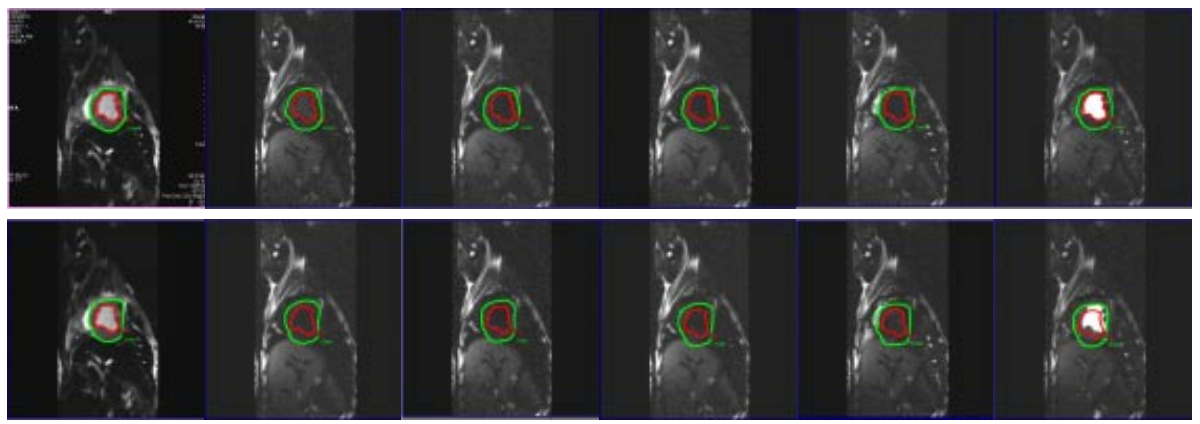

Fig. 2. Time sequence of MR perfusion obtained for a heavily sedated dog. The first row of images show the result obtained using pixel intensity based MI algorithm. For these results only whole pixel shifts are being estimated. The second row of images shows the results obtained using MI based strategy with sub-pixel accuracy.

However, results from Fig 1 showed that sub-pixel shifts might add-up for a big drifts. Therefore we implemented mutual information based strategy where we are estimating sub-pixel shifts. The second row of images in Fig.2] show the results obtained where we are estimating sub-pixel shifts. Note that while estimating the sub-pixel shifts lead to drifts in the estimated myocardium. This happens due to the fact that mutual information $I(f, y)$ between two random variables $f$ and $y$, given by $I(f, y)=H(f)+H(y)-H(f, y)$ is while trying to minimize the joint entropy $H(f, y)$ is also trying to maximize the marginal entropy $H(y)$. For estimating sub-pixel shifts, interpolation of the pixel intensities is required. Interpolation is effectively smoothing the image and hence reduces the marginal entropy $H(y)$. To compensate for the reduction of marginal entropy due to smoothing, the algorithm shifts the optimal position where there is more 


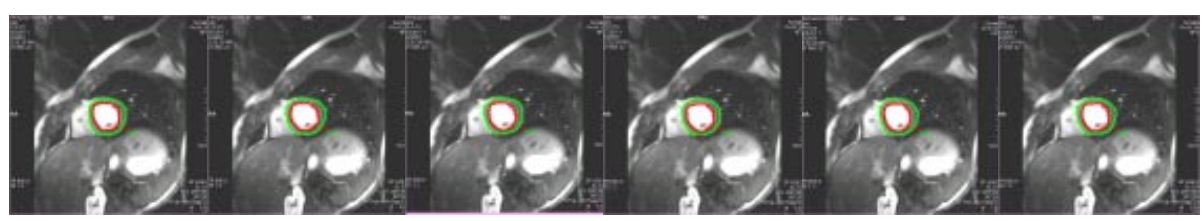

Fig. 3. Results obtained using our proposed integrated registration algorithm on a real patient MR perfusion sequence. Note again we are estimating only whole pixel shifts.

variations in pixel intensities. This conclusion was supported by the fact that we noticed shifts of the contours only when there was no contrast in the images. When there was contrast in the images, and hence already high marginal entropy $H(y)$, there were no spurious shifts of the contours. Since estimating sub-pixel shifts can lead to spurious drifts in the data where contrast is rapidly changing, we decided to estimate only whole pixel shifts. However, to account for drifts due to sub-pixel shifts, we incorporated a second channel of information in the form of edge information to pull the contours to myocardium.

The second row of images in Fig[1] shows the results obtained using our combined strategy. Notice that while we are estimating only the whole pixel shifts, the edge information in our registration framework pulls the contours every few frames to the right position so that the sub-pixel shifts do not add up.

Fig. 3 shows results obtained using our integrated registration approach on the MR perfusion sequence of a real patient. Notice that using our integrated approach, we are able to segment the myocardium reliably in the complete sequence. An important point to make is that we cannot just use the edge information in the template correlation due to rapidly changing contrast in the MR perfusion sequence. In the sequence of images

\section{Discussion}

Results from Fig.1 showed that the registration results obtained while estimating only whole pixel shifts might not be sufficient if sub-pixel shifts are present in the image sequence. However, estimation of sub-pixel shifts can lead to spurious drifts in the sequence of images if there are images in the sequence with very low contrast, as shown in Fig, 2 These spurious drifts occur due to the fact the while estimating sub-pixel shifts, the interpolation reduces the marginal entropy. Thus we devised a strategy, which while estimating only whole pixel shifts can account for sub-pixel shifts. To do this we proposed an integrated registration framework which integrated two channels of information, the pixel intensities and the local gradient, in one unifying registration framework. Notice that since we are estimating only whole pixel shifts, there will be sub-pixel errors in the estimated myocardial position but the edge term will pull the contours to the right location ever so often in the sequence such that the sub-pixel shifts do not add up. Our results seem to bring out explicitly the fact that care should 
be taken while trying to estimate sub-pixel shifts using MI based registration strategy.

As was mentioned earlier in the paper, while every effort is made during acquisition of MR perfusion sequence to obtain each image during the phase in the heart cycle, the shape of the heart changes locally. This is an issue which was not addressed in this paper. As a future work we plan to apply local deformations to the estimated contours to more accurately segment the changing heart shape.

\section{References}

1. N. Al-Saadi et al. Noninvasive detection of myocardial ischemia from perfusion reserve based on cardiovascular magnetic resonance. Circulation, 101, 2000.

2. M. Breeuwer et al. Automatic detection of the myocardial boundaries of the right and left ventricle in MR cardio perfusion scans. Proc. SPIE Med. Imag., Feb. 2001.

3. A. Collignon, F. Maes, et al. Automated multimodality image registration using information theory. Info. Proc. in Med. Imag. (IPMI), pages 263-274, 1995.

4. S. Geman and D. Geman. Stochastic relaxation, gibbs distributions and the bayesian restoration of images. IEEE Trans. PAMI, 6(6):721-741, Nov. 1984.

5. A. Hamadeh et al. A unified approach to 3D-2D registration and 2D images segmentation. In H. U. Lemke, K. Inamura, C. C. Jaffe, and M. W. Vannier, editors, Computer assisted radiology, pages 1191-1196, 1995. Springer-Verlag.

6. J.J. Hopfield. Neurons with graded response have computational properties like those of two-state neurons. Proc. Natl. Acad. Sci., 81:3088-3092, 1984.

7. S.Z. Li. Markov Random Field Modeling in Computer Vision. Springer, 1995.

8. Jerosch H. M. et al. MR first pass imaging: quantitative assessment of transmural perfusion and collateral flow. Int. J. Card. Imaging, 13:205-218, 1997.

9. J. Pluim et al. Image registration by maximization of combined mutual information and gradient information. IEEE Trans. Med. Imag., 19(8), 2000.

10. Alexis Roche et al. Rigid registration of 3D ultrasound with MR images: a new approach combining intensity and gradient information. IEEE TMI, 2001.

11. Go RT et al. A prospective comparison of rubidium 82 PET and thallium 201 SPECT myocardial perfusion imaging utilizing a single dipyridamole stress in the diagnosis of coronary artery disease. J. Nucl. Med., 31:1899-1905, 1990.

12. M. Schwaiger. Myocardial perfusion imaging with PET. J. Nucl. Med., 35:693-698, 1994.

13. L. Spreeuwers et al. Automatic detection of myocardial boundaries in MR cardio perfusion images. MICCAI, 2001. M88.

14. P. Viola and W. M. Wells. Alignment by maximization of mutual information. Fifth Int. Conf. on Computer Vision, pages 16-23, 1995.

15. N. Wilke et al. Myocardial perfusion reserve: assessment with multisection, quantitative, first pass MR imaging. Radiology, 204:373-384, 1997.

16. A. Yullie. Energy functions for early vision and analog networks. Biol. Cybern, 61:115-124, 1989 . 